\title{
SOME RESULTS ON RICCI-BOURGUIGNON SOLITONS AND ALMOST SOLITONS
}

\author{
SHUBHAM DWIVEDI
}

\begin{abstract}
We prove some results for the solitons of the Ricci-Bourguignon flow, generalizing the corresponding results for Ricci solitons. Taking motivation from Ricci almost solitons, we then introduce the notion of RicciBourguignon almost solitons and prove some results about them which generalize previous results for Ricci almost solitons. We also derive integral formulas for compact gradient Ricci-Bourguignon solitons and compact gradient Ricci-Bourguignon almost solitons. Finally, using the integral formula, we show that a compact gradient Ricci-Bourguignon almost soliton is isometric to a Euclidean sphere if it has constant scalar curvature or its associated vector field is conformal.
\end{abstract}

2020 Mathematics Subject Classification : 53C20; 53C21; 53E20.

\section{INTRODUCTION}

Ricci solitons play a major role in Ricci flow where they correspond to self-similar solutions of the flow. Thus, given a geometric flow, it is natural to study the solitons associated to that flow. A family of metrics $g(t)$ on an $n$-dimensional Riemannian manifold $\left(M^{n}, g\right)$ is said to evolve by the Ricci-Bourguignon flow (RB flow for short) if $g(t)$ satisfies the following evolution equation

$$
\frac{\partial g}{\partial t}=-2(\operatorname{Ric}-\rho R g)
$$

where Ric is the Ricci tensor of the metric, $R$ is the scalar curvature and $\rho \in \mathbb{R}$ is a constant. The flow in equation (1.1) was first introduced by Jean-Pierre Bourguignon [Bou81], building on some unpublished work of Lichnerowicz and a paper of Aubin [Aub70]. We note that (1.1) is precisely the Ricci flow for $\rho=0$. In particular, the right hand side of the evolution equation (1.1) is of special interest for different values of $\rho$, for example

- $\rho=\frac{1}{2}$, the Einstein tensor Ric $-\frac{R}{2} g$.

- $\rho=\frac{1}{n}$, the traceless Ricci tensor Ric $-\frac{R}{n} g$.

- $\rho=\frac{1}{2(n-1)}$, the Schouten tensor Ric $-\frac{R}{2(n-1)} g$.

- $\rho=0$, the Ricci tensor Ric.

A systematic study of the parabolic theory of the RB flow was initiated in $\left[\mathrm{CCD}^{+} 17\right]$. In that paper, the authors proved, along with many other results, the short time existence of the flow (1.1) on any closed $n$ dimensional manifold starting with an arbitrary initial metric $g_{0}$ for $\rho<\frac{1}{2(n-1)}$. Just like the Ricci flow case, we make the following

Definition 1.1. A Ricci-Bourguignon soliton (RB soliton for short) is a Riemannian manifold $\left(M^{n}, g\right)$ endowed with a vector field $X$ on $M$ that satisfies

$$
R_{i j}+\frac{1}{2}\left(\mathcal{L}_{X} g\right)_{i j}=\lambda g_{i j}+\rho R g_{i j}
$$

where $\mathcal{L}_{X} g$ denotes the Lie derivative of the metric $g$ with respect to the vector field $X$ and $\lambda \in \mathbb{R}$ is a constant.

When $X=\nabla f$ for some smooth $f: M \rightarrow \mathbb{R}$, then $(M, g)$ is called a gradient $\mathrm{RB}$ soliton. The soliton is called

(1) expanding when $\lambda<0$,

(2) steady when $\lambda=0$,

(3) shrinking when $\lambda>0$.

$\mathrm{RB}$ solitons correspond to self-similar solutions of the RB flow. An RB soliton is called trivial if $X$ is a Killing vector field, i.e., $\mathcal{L}_{X} g=0$. We remark that even though the short time existence result for the flow (1.1) is for $\rho<\frac{1}{2(n-1)}$, any value of $\rho$ is possible for the considerations of self-similar solutions of the flow. 
Gradient RB solitons were studied in detail, for example in [CM16] and [CMM15], where the authors called them gradient $\rho$-Einstein solitons. Various classification and rigidity results about gradient RB solitons were proved in those papers and we refer the reader to those papers for precise statements and proofs of the results.

The notion of Ricci almost solitons was introduced in [PRRS11], where the authors modified the definition of a Ricci soliton by considering the parameter $\lambda$ in the definition of a Ricci soliton to be a function rather than a constant. Motivated by the Ricci flow case, we make the following

Definition 1.2. A Riemannian manifold $\left(M^{n}, g\right)$ is a Ricci-Bourguignon almost soliton (RB almost soliton for short) if there is a vector field $X$ and a soliton function $\lambda: M \rightarrow \mathbb{R}$ satisfying

$$
\operatorname{Ric}+\frac{1}{2} \mathcal{L}_{X} g=\lambda g+\rho R g
$$

An $\mathrm{RB}$ almost soliton is called a gradient $\mathrm{RB}$ almost soliton if $X=\nabla f$ for some smooth function $f$ on $M$ and is expanding, steady or shrinking if $\lambda<0, \lambda=0$ or $\lambda>0$, respectively. We note that if $X$ is a Killing vector field, then a $R B$ almost soliton is just a $R B$ soliton as it forces $\lambda$ to be a constant.

Recall that a vector field $Y$ on a Riemannian manifold $(M, g)$ is called a conformal vector field if there exists a function $\psi: M \rightarrow \mathbb{R}$ such that

$$
\mathcal{L}_{Y} g=2 \psi g \text {. }
$$

The function $\psi$ is sometimes referred as the potential of the vector field $Y$. The conformal vector field is nontrivial if $\psi \neq 0$. If $\psi=0$, then $Y$ is called a Killing vector field.

Some characterization results for compact Ricci and Ricci almost solitons were obtained in [ABR11] and [BR12] respectively. The goal of the present paper is to generalize the results obtained in those papers to RB and RB almost solitons. More precisely, in $\S 3$ we prove the following theorems.

Theorem 1.3. Let $\left(M^{n}, g, X, \lambda, \rho\right), n \geq 3$, be a $R B$ soliton and suppose that the vector field $X$ is a conformal vector field.

(1) If $M$ is compact, then $X$ is a Killing vector field and hence $\left(M^{n}, g, X, \lambda, \rho\right)$ is a trivial RB soliton.

(2) There is no nontrivial, complete noncompact $R B$ soliton $\left(M^{n}, g, X, \lambda, \rho\right)$ with conformal vector field $X$.

This generalizes Theorem 3 in [ABR11] and characterizes compact RB solitons when $X$ is a conformal vector field. The following corollary gives a lower bound for the first eigenvalue of the Laplacian on a compact RB soliton when $X$ is a conformal vector field and generalizes Theorem 4 in [ABR11].

Corollary 1.4. Let $\left(M^{n}, g, X, \lambda, \rho\right)$ be a compact $R B$ soliton with $X$ a conformal vector field. If $n \geq 3$ and $\lambda+\rho R>0$, then the first eigenvalue $\lambda_{1}$ of the Laplacian satisfies $\lambda_{1} \geq(\lambda+\rho R) \frac{n}{n-1}$. Moreover, equality occurs if and only if $M^{n}$ is isometric to a Euclidean sphere.

The next theorem characterizes compact RB almost solitons with $X$ a conformal vector field and generalizes Theorem 2 in [BR12].

Theorem 1.5. Let $\left(M^{n}, g, X, \lambda, \rho\right), n \geq 3$, be a compact $R B$ almost soliton. If $X$ is a nontrivial conformal vector field, then $M^{n}$ is isometric to a Euclidean sphere.

Example 1.6. Consider the Euclidean sphere $\left(S^{n}, g_{\text {round }}, X, \lambda\right)$, where $g_{\text {round }}$ is the round metric on $S^{n}, X$ is the projection of a non-zero constant vector field $\bar{X}$ on $\mathbb{R}^{n+1}$, over $S^{n}$ and $\lambda=1-\rho(n-1) \operatorname{div} X$, with $\rho \in \mathbb{R}$ a constant. Then $X$ is a conformal vector field on $S^{n}$ and is not a Killing vector field. Since Ric $=(n-1) g_{\text {round }}$ for $S^{n},\left(S^{n}, g_{\text {round }}, X, \lambda\right)$ is a $\mathrm{RB}$ almost soliton.

Example 1.7. Let $M=I \times_{h} \Sigma$ be the $h$-warped product of the real interval $I \subset \mathbb{R}$ with $0 \in I$, and the Riemannian manifold $\left(\Sigma, g_{\Sigma}\right)$ with $\operatorname{dim} \Sigma=n$. That is, $M$ is an $(n+1)$-dimensional product manifold $I \times \Sigma$ with the metric

$$
g_{M}=d t^{2}+h^{2} g_{\Sigma},
$$

where $t$ is a global parameter on $I$ and $h$ is a positive function on $I$. Suppose $\Sigma$ is an Einstein manifold with $\operatorname{Ric}_{\Sigma}=-(n-1) a$ with $a<0$. Following [PRRS11, Example 2.5], define a function $f(x, t)=f(t)$ by

$$
f(t)=B+\int_{0}^{t} h(s)\left[A+(n-1) \int_{0}^{s} \frac{h^{\prime \prime} h-\left(h^{\prime}\right)^{2}-a}{h^{3}} d x\right] d s
$$


for some constants $A, B \in \mathbb{R}$. Also define, $\lambda(x, t)=\lambda(t)$ by

$$
\lambda(t)=-(n-1)\left[\frac{\left(h^{\prime}\right)^{2}+a}{h^{2}}-a n \rho\right]-\frac{h^{\prime \prime}}{h}+h^{\prime}\left[A+(n-1) \int_{0}^{s} \frac{h^{\prime \prime} h-\left(h^{\prime}\right)^{2}-a}{h^{3}} d x\right],
$$

where $\rho \in \mathbb{R}$ is a constant. It follows from [PRRS11] that $\left(M, g_{M}, f^{\prime} \frac{\partial}{\partial t}, \lambda, \rho\right)$ is a RB almost soliton, where $f$ is given by (1.4) and $\lambda$ is given by (1.5).

The next theorem generalizes Theorem 3 in [BR12] obtained for compact Ricci almost solitons, which is the case when $\rho=0$.

Theorem 1.8. Let $\left(M^{n}, g, X, \lambda, \rho\right), n \geq 3$, be a compact $R B$ almost soliton. If $\rho \neq \frac{1}{n}$ and

$$
\int_{M}\left[\operatorname{Ric}(X, X)+\frac{n \rho}{n \rho-1} \nabla_{X} \operatorname{div} X-2 \rho g(\nabla R, X)-\frac{(n(2 \rho+1)-2)}{n \rho-1} g(\nabla \lambda, X)\right] d v \leq 0,
$$

then $X$ is a Killing vector field and $M^{n}$ is a trivial $R B$ soliton.

Since every $\mathrm{RB}$ almost soliton is also a $\mathrm{RB}$ soliton for constant $\lambda$, hence using $\nabla \lambda=0$, we get the following corollary for compact RB solitons.

Corollary 1.9. Let $\left(M^{n}, g, X, \lambda, \rho\right), n \geq 3$, be a compact $R B$ soliton. If $\rho \neq \frac{1}{n}$ and

$$
\int_{M}\left[\operatorname{Ric}(X, X)+\frac{n \rho}{(n \rho-1)} \nabla_{X} \operatorname{div} X-2 \rho g(\nabla R, X)\right] d v \leq 0
$$

then $X$ is a Killing vector field and $M^{n}$ is a trivial $R B$ soliton.

Remark 1.10. Corollary 1.9 is an analog of Theorem 1.1 in [PW09], which was for the case of compact Ricci solitons. We obtain Petersen-Wylie's result from our result by taking $\rho=0$. In fact, the condition in (1.7) is analogous to the condition in [PW09, Theorem 1.1], which is obtained when $\rho=0$ in (1.7).

Finally, we obtain integral formulas for compact gradient RB almost solitons, generalizing a corresponding result for compact gradient Ricci almost solitons from [BR12].

Theorem 1.11. Let $\left(M^{n}, g, \nabla f, \lambda, \rho\right)$ be a compact gradient $R B$ almost soliton. Then

$$
\int_{M}\left|\nabla^{2} f-\frac{\Delta f}{n} g\right|^{2} d v=\frac{(n-2)}{2 n} \int_{M} g(\nabla R, \nabla f) d v
$$

and

$$
\int_{M}\left|R i c-\frac{R}{n} g\right|^{2} d v=\frac{(n-2)}{2 n} \int_{M} g(\nabla R, \nabla f) d v
$$

As an application of the previous theorem, we provide some conditions for a compact gradient RB almost soliton to be isometric to a Euclidean sphere.

Corollary 1.12. A nontrivial compact gradient $R B$ almost soliton $\left(M^{n}, g, \nabla f, \lambda, \rho\right), n \geq 3$, is isometric to a Euclidean sphere if any of the following assertions hold:

(1) $M^{n}$ has constant scalar curvature.

(2) $\int_{M} g(\nabla R, \nabla f) d v \leq 0$.

(3) $M^{n}$ is a homogenous manifold.

The paper is organized as follows. In $\S 2$, we state and prove some identities for RB solitons and RB almost solitons which will be used to prove the main results. In $\S 3$, we prove the main theorems and their corollaries.

Acknowledgments. The author would like to thank his advisor Spiro Karigiannis for his constant encouragement and advice. The author is also grateful to the anonymous referee for various useful remarks and suggestions which have improved the quality of the paper. 


\section{Preliminaries}

In this section, we prove some general results about $\mathrm{RB}$ solitons and almost solitons. The proofs of some of these results in the compact gradient case can also be found in [CM16] or [CMM15]. Let us first recall the Ricci identity for a $(0,2)$-tensor $\alpha$ :

$$
\nabla_{i} \nabla_{j} \alpha_{k l}-\nabla_{j} \nabla_{i} \alpha_{k l}=-R_{i j k m} \alpha_{m l}-R_{i j l m} \alpha_{k m},
$$

where $R_{i j k l}$ is the Riemann curvature tensor. The Ricci curvature is obtained from the Riemann curvature tensor by contracting on the first and last index

$$
R_{i j}=g^{k l} R_{k i j l}
$$

and the contracted second Bianchi identity is

$$
\nabla_{i} R_{i j}=\frac{1}{2} \nabla_{j} R
$$

We start with the following

Proposition 2.1. Let $\left(M^{n}, g, \nabla f, \lambda, \rho\right)$ be a gradient $R B$ almost soliton. Then the following identities hold

$$
\begin{aligned}
(1-n \rho) R+\Delta f= & n \lambda . \\
(1-2 \rho(n-1)) \nabla_{i} R= & 2 R_{i l} \nabla_{l} f+2(n-1) \nabla_{i} \lambda . \\
\nabla_{j} R_{i k}-\nabla_{k} R_{i j}= & R_{j k i l} \nabla_{l} f+\rho\left(\nabla_{j} R g_{i k}-\nabla_{k} R g_{i j}\right) \\
& +\left(\nabla_{j} \lambda g_{i k}-\nabla_{k} \lambda g_{i j}\right) . \\
\nabla_{i}\left[(1-2 \rho(n-1)) R+|\nabla f|^{2}-2(n-1) \lambda\right]= & (2 \rho R+2 \lambda) \nabla_{i} f .
\end{aligned}
$$

Proof. For a gradient RB almost soliton we have

$$
R_{i j}+\nabla_{i} \nabla_{j} f=\lambda g_{i j}+\rho R g_{i j} .
$$

Taking the trace of the above equation gives (2.1). Next, taking the covariant derivative of (2.1) with respect to an orthonormal frame gives

$$
(1-n \rho) \nabla_{i} R+\nabla_{i} \nabla_{j} \nabla_{j} f=n \nabla_{i} \lambda .
$$

Commuting the covariant derivatives and using the contracted second Bianchi identity, we obtain

$$
\begin{aligned}
(1-n \rho) \nabla_{i} R & =-\nabla_{j} \nabla_{i} \nabla_{j} f+R_{i l} \nabla_{l} f+n \nabla_{i} \lambda \\
& =-\nabla_{j}\left(-R_{i j}+\lambda g_{i j}+\rho R g_{i j}\right)+R_{i l} \nabla_{l} f+n \nabla_{i} \lambda \\
& =\frac{1}{2} \nabla_{i} R-\rho \nabla_{i} R-\nabla_{i} \lambda+R_{i l} \nabla_{l} f+n \nabla_{i} \lambda
\end{aligned}
$$

and hence

$$
\left(\frac{1}{2}-\rho(n-1)\right) \nabla_{i} R=R_{i l} \nabla_{l} f+(n-1) \nabla_{i} \lambda,
$$

which proves (2.2).

For proving (2.3), we use (2.5) and commute the covariant derivatives to get

$$
\begin{aligned}
\nabla_{j} R_{i k}-\nabla_{k} R_{i j}= & \left(\nabla_{k} \nabla_{i} \nabla_{j} f-\nabla_{j} \nabla_{i} \nabla_{k} f\right)+\rho\left(\nabla_{j} R g_{i k}-\nabla_{k} R g_{i j}\right) \\
& +\left(\nabla_{j} \lambda g_{i k}-\nabla_{k} \lambda g_{i j}\right) \\
= & \left(\nabla_{k} \nabla_{j} \nabla_{i} f-\nabla_{j} \nabla_{k} \nabla_{i} f\right)+\rho\left(\nabla_{j} R g_{i k}-\nabla_{k} R g_{i j}\right) \\
& +\left(\nabla_{j} \lambda g_{i k}-\nabla_{k} \lambda g_{i j}\right) \\
= & R_{j k i l} \nabla_{l} f+\rho\left(\nabla_{j} R g_{i k}-\nabla_{k} R g_{i j}\right)+\left(\nabla_{j} \lambda g_{i k}-\nabla_{k} \lambda g_{i j}\right) .
\end{aligned}
$$

Finally, from $(2.2)$ we get

$$
\begin{aligned}
(1-2 \rho(n-1)) \nabla_{i} R & =2 \nabla_{l} f\left(-\nabla_{i} \nabla_{l} f+\lambda g_{i l}+\rho R g_{i l}\right)+2(n-1) \nabla_{i} \lambda \\
& =-2 \nabla_{l} f \nabla_{i} \nabla_{l} f+2 \lambda \nabla_{i} f+2 \rho R \nabla_{i} f+2(n-1) \nabla_{i} \lambda \\
& =-\nabla_{i}\left|\nabla_{l} f\right|^{2}+2 \lambda \nabla_{i} f+2 \rho R \nabla_{i} f+2(n-1) \nabla_{i} \lambda,
\end{aligned}
$$

so we get

$$
\nabla_{i}\left[(1-2 \rho(n-1)) R+|\nabla f|^{2}-2(n-1) \lambda\right]=(2 \rho R+2 \lambda) \nabla_{i} f
$$

which proves (2.4). 
Remark 2.2. The analogous identities for gradient RB solitons $\left(M^{n}, g, \nabla f, \lambda, \rho\right)$ are

$$
\begin{aligned}
(1-n \rho) R+\Delta f & =n \lambda . \\
(1-2 \rho(n-1)) \nabla_{i} R & =2 R_{i l} \nabla_{l} f . \\
\nabla_{j} R_{i k}-\nabla_{k} R_{i j} & =R_{j k i l} \nabla_{l} f+\rho\left(\nabla_{j} R g_{i k}-\nabla_{k} R g_{i j}\right) . \\
\nabla_{i}\left[(1-2 \rho(n-1)) R+|\nabla f|^{2}-2 \lambda f\right] & =2 \rho R \nabla_{i} f .
\end{aligned}
$$

The proofs of these identities are special cases of the previous result as $\nabla \lambda=0$.

We recall the following lemma from [PW09, Lemma 2.1].

Lemma 2.3. Let $X$ be a vector field on a Riemannian manifold $\left(M^{n}, g\right)$. Then

$$
\operatorname{div}\left(\mathcal{L}_{X} g\right)(X)=\frac{1}{2} \Delta|X|^{2}-|\nabla X|^{2}+\operatorname{Ric}(X, X)+\nabla_{X} \operatorname{div} X
$$

When $X=\nabla f$ and $Z$ is any vector field, then

$$
\operatorname{div}\left(\mathcal{L}_{\nabla f} g\right)(Z)=2 \operatorname{Ric}(Z, \nabla f)+2 \nabla_{Z} \operatorname{div} \nabla f .
$$

We use the preceding lemma to prove the following

Lemma 2.4. Let $\left(M^{n}, g, X, \lambda, \rho\right)$ be a $R B$ almost soliton. Then

$$
\begin{aligned}
\frac{(1-n \rho)}{2} \Delta|X|^{2}= & (1-n \rho)|\nabla X|^{2}+(n \rho-1) \operatorname{Ric}(X, X)+n \rho \nabla_{X} \operatorname{div} X \\
& +2 \rho(1-n \rho) g(\nabla R, X)-(n(2 \rho+1)-2) g(\nabla \lambda, X)
\end{aligned}
$$

and

$$
\begin{aligned}
\frac{(1-n \rho)}{2}\left(\Delta-\nabla_{X}\right)|X|^{2}= & (1-n \rho)|\nabla X|^{2}+\lambda(n \rho-1)|X|^{2}+\rho(n \rho-1) R|X|^{2} \\
& +n \rho \nabla_{X} \operatorname{div} X+2 \rho(1-n \rho) g(\nabla R, X) \\
& -(n(2 \rho+1)-2) g(\nabla \lambda, X) .
\end{aligned}
$$

Proof. We first notice that (1.3) gives

$$
2 \operatorname{div} \operatorname{Ric}+\operatorname{div}\left(\mathcal{L}_{X} g\right)=2 \nabla \lambda+2 \rho \nabla R .
$$

Taking the trace of $(1.3)$ gives $(1-n \rho) R+\operatorname{div} X=n \lambda$ and thus

$$
(1-n \rho) \nabla_{X} R+\nabla_{X}(\operatorname{div} X)=n \nabla_{X} \lambda \text {. }
$$

So, using (2.13), (2.17), (2.18) and the contracted second Bianchi identity, we get

$$
\begin{aligned}
\nabla_{X}(\operatorname{div} X)= & (n \rho-1) \nabla_{X} R+n g(\nabla \lambda, X) \\
= & 2(n \rho-1) \operatorname{div} \operatorname{Ric}(X)+n g(\nabla \lambda, X) \\
= & -(n \rho-1) \operatorname{div}\left(\mathcal{L}_{X} g\right)(X)+2 \rho(n \rho-1) g(\nabla R, X)+2(n \rho-1) g(\nabla \lambda, X) \\
& +n g(\nabla \lambda, X) \\
= & (1-n \rho)\left(\frac{1}{2} \Delta|X|^{2}-|\nabla X|^{2}+\operatorname{Ric}(X, X)+\nabla_{X} \operatorname{div} X\right) \\
& +2 \rho(n \rho-1) g(\nabla R, X)+(n(2 \rho+1)-2) g(\nabla \lambda, X) \\
= & \frac{(1-n \rho)}{2} \Delta|X|^{2}-(1-n \rho)|\nabla X|^{2}+(1-n \rho) \operatorname{Ric}(X, X) \\
& +(1-n \rho) \nabla_{X} \operatorname{div} X+2 \rho(n \rho-1) g(\nabla R, X)+(n(2 \rho+1)-2) g(\nabla \lambda, X),
\end{aligned}
$$

which gives

$$
\begin{aligned}
\frac{(1-n \rho)}{2} \Delta|X|^{2}= & (1-n \rho)|\nabla X|^{2}+(n \rho-1) \operatorname{Ric}(X, X)+n \rho \nabla_{X} \operatorname{div} X \\
& +2 \rho(1-n \rho) g(\nabla R, X)-(n(2 \rho+1)-2) g(\nabla \lambda, X),
\end{aligned}
$$

thus proving (2.15). 
Using (1.3) to write $\operatorname{Ric}(X, X)=-\frac{1}{2}\left(\mathcal{L}_{X} g\right)(X, X)+\lambda|X|^{2}+\rho R|X|^{2}$ in (2.15), we get

$$
\begin{aligned}
\frac{(1-n \rho)}{2} \Delta|X|^{2}= & (1-n \rho)|\nabla X|^{2}+(n \rho-1)\left(-\frac{1}{2}\left(\mathcal{L}_{X} g\right)(X, X)+\lambda|X|^{2}+\rho R|X|^{2}\right) \\
& +n \rho \nabla_{X} \operatorname{div} X+2 \rho(1-n \rho) g(\nabla R, X)-(n(2 \rho+1)-2) g(\nabla \lambda, X) \\
= & (1-n \rho)|\nabla X|^{2}+\frac{(1-n \rho)}{2} \nabla_{X}|X|^{2}+\lambda(n \rho-1)|X|^{2}+\rho(n \rho-1) R|X|^{2} \\
& +n \rho \nabla_{X} \operatorname{div} X+2 \rho(1-n \rho) g(\nabla R, X)-(n(2 \rho+1)-2) g(\nabla \lambda, X),
\end{aligned}
$$

which gives

$$
\begin{aligned}
\frac{(1-n \rho)}{2}\left(\Delta-\nabla_{X}\right)|X|^{2}= & (1-n \rho)|\nabla X|^{2}+\lambda(n \rho-1)|X|^{2}+\rho(n \rho-1) R|X|^{2} \\
& +n \rho \nabla_{X} \operatorname{div} X+2 \rho(1-n \rho) g(\nabla R, X) \\
& -(n(2 \rho+1)-2) g(\nabla \lambda, X),
\end{aligned}
$$

proving (2.16).

If we consider the diffusion operator $\Delta_{X}=\Delta-\nabla_{X}$, then from the previous lemma with $X=\nabla f$ and $\Delta_{f}=\Delta-\nabla_{\nabla f}$, we obtain the following corollary.

Corollary 2.5. For a gradient $R B$ almost soliton $\left(M^{n}, g, \nabla f, \lambda, \rho\right)$, we have

$$
\begin{aligned}
\frac{(1-n \rho)}{2} \Delta_{f}|\nabla f|^{2}= & (1-n \rho)\left|\nabla^{2} f\right|^{2}+\lambda(n \rho-1)|\nabla f|^{2}+\rho(n \rho-1) R|\nabla f|^{2} \\
& +n \rho \nabla_{\nabla f}(\Delta f)+2 \rho(1-n \rho) g(\nabla R, \nabla f) \\
& -(n(2 \rho+1)-2) g(\nabla \lambda, \nabla f) .
\end{aligned}
$$

Remark 2.6. The analogs of (2.15) and (2.16) for a RB soliton $\left(M^{n}, g, X, \lambda, \rho\right)$ are

$$
\begin{aligned}
\frac{(1-n \rho)}{2} \Delta|X|^{2}= & (1-n \rho)|\nabla X|^{2}+(n \rho-1) \operatorname{Ric}(X, X)+n \rho \nabla_{X} \operatorname{div} X \\
& +2 \rho(1-n \rho) g(\nabla R, X)
\end{aligned}
$$

and

$$
\begin{aligned}
\frac{(1-n \rho)}{2}\left(\Delta-\nabla_{X}\right)|X|^{2}= & (1-n \rho)|\nabla X|^{2}+\lambda(n \rho-1)|X|^{2}+\rho(n \rho-1) R|X|^{2} \\
& +n \rho \nabla_{X} \operatorname{div} X+2 \rho(1-n \rho) g(\nabla R, X) .
\end{aligned}
$$

The proofs are special cases of the proof of Lemma 2.4 with $\nabla \lambda=0$.

\section{Proofs of the Results}

We start this section by proving the following lemma which will be used in the proofs of Theorem 1.3 and Theorem 1.5.

Lemma 3.1. Let $\left(M^{n}, g, X, \lambda, \rho\right), n \geq 3$, be a $R B$ almost soliton. If $X$ is a nontrivial conformal vector field with $\mathcal{L}_{X} g=2 \psi g$, then $R$ and $\lambda-\psi$ are constant.

Proof. The soliton equation is

$$
R_{i j}+\frac{1}{2}\left(\mathcal{L}_{X} g\right)_{i j}=\lambda g_{i j}+\rho R g_{i j},
$$

where $\lambda: M \rightarrow \mathbb{R}$ is a function. If $X$ is a nontrivial conformal vector field, then we have

$$
\mathcal{L}_{X} g=2 \psi g,
$$

for some function $\psi: M \rightarrow \mathbb{R}, \psi \neq 0$. So (3.1) becomes

$$
R_{i j}=(\lambda-\psi+\rho R) g_{i j} .
$$

Taking the divergence of (3.3), we get

$$
\nabla_{i} R_{i j}=\nabla_{i}(\lambda-\psi+\rho R) g_{i j},
$$


which implies

$$
\left(\frac{1}{2}-\rho\right) \nabla_{j} R=\nabla_{j}(\lambda-\psi)
$$

On the other hand, tracing (3.3) and taking the covariant derivative, we get

$$
(1-n \rho) \nabla_{j} R=n \nabla_{j}(\lambda-\rho) .
$$

So from (3.4) and (3.5), we get

$$
(1-n \rho) \nabla_{j} R=n\left(\frac{1}{2}-\rho\right) \nabla_{j} R .
$$

Thus, if $M$ is connected, then $R$ is constant and hence $\lambda-\psi$ is constant.

Remark 3.2. If $\left(M^{n}, g, X, \lambda, \rho\right), n \geq 3$, is a $\mathrm{RB}$ soliton and $X$ is a conformal vector field with $\mathcal{L}_{X} g=2 \psi g$ for some function $\psi: M \rightarrow \mathbb{R}$, then the proof of Lemma 3.1 shows that $R$ and $\psi$ are constant as in this case $\nabla \lambda=0$.

We prove Theorem 1.3 which we restate here.

Theorem 3.3. Let $\left(M^{n}, g, X, \lambda, \rho\right), n \geq 3$, be a $R B$ soliton and suppose that the vector field $X$ is a conformal vector field.

(1) If $M$ is compact, then $X$ is a Killing vector field and hence $\left(M^{n}, g, X, \lambda, \rho\right)$ is a trivial RB soliton.

(2) There is no nontrivial, complete noncompact $R B$ soliton $\left(M^{n}, g, X, \lambda, \rho\right)$ with conformal vector field $X$.

Proof. Suppose $X$ is a conformal vector field with potential $\psi: M \rightarrow \mathbb{R}$, i.e.,

$$
\mathcal{L}_{X} g=2 \psi g,
$$

then from Remark 3.2 we know that $R$ and $\psi$ are constant.

Taking the trace of (3.7), we get

$$
2 \operatorname{div} X=2 n \psi,
$$

which upon integration over compact $M$ gives

$$
0=\int_{M} 2 \operatorname{div} X d v=2 n \operatorname{Vol}(M) \psi,
$$

which implies that $\psi=0$. So $X$ is a Killing vector field and hence $\left(M^{n}, X, g, \lambda, \rho\right)$ is a trivial RB soliton.

If $M$ is noncompact and a gradient $\mathrm{RB}$ soliton with $X=\nabla f$, then $X$ being conformal implies

$$
\nabla_{i} \nabla_{j} f=\psi g_{i j}
$$

and by Remark 3.2, $\psi$ is constant. If $\psi=0$, then $X$ is a Killing vector field and $M$ is a trivial RB soliton. If $\psi \neq 0$, then from [Tas65, Theorem 2], we conclude that $M^{n}$ is isometric to the Euclidean space.

Next we prove Corollary 1.4 .

Proof. Since $M^{n}$ is compact, we know from Theorem 1.3 that $X$ is a Killing vector field and hence we have Ric $=(\lambda+\rho R) g$. So we can apply a classical theorem due to Lichnerowicz [Lic58], which states that if Ric $\geq k$, where $k>0$ is a constant, then the first eigenvalue of the Laplacian $\lambda_{1}$ satisfies $\lambda_{1} \geq \frac{n}{n-1} k$. So we get

$$
\lambda_{1} \geq(\lambda+\rho R) \frac{n}{n-1} .
$$

Moreover, for the equality case, we can apply Obata's theorem [Oba62], to conclude that equality occurs in the above inequality if and only if $M^{n}$ is isometric to a Euclidean sphere of constant curvature $\frac{(\lambda+\rho R)}{n-1}$.

We now prove Theorem 1.5, which we restate here.

Theorem 3.4. Let $\left(M^{n}, g, X, \lambda, \rho\right), n \geq 3$, be a compact $R B$ almost soliton. If $X$ is a nontrivial conformal vector field, then $M^{n}$ is isometric to a Euclidean sphere. 
Proof. Suppose $X$ is a nontrivial conformal vector field with potential function $\psi: M \rightarrow \mathbb{R}$, i.e.,

$$
\mathcal{L}_{X} g=2 \psi g,
$$

with $\psi \neq 0$. Since $\left(M^{n}, g, X, \lambda, \rho\right)$ is a compact RB almost soliton with $n \geq 3$, Lemma 3.1 tells us that $R$ and $\lambda-\psi$ are constant. So from Lemma 2.3 in [Yan70, pg.52], we conclude that $R \neq 0$ or else $\psi$ would be 0 . Taking the Lie derivative of (3.3), we get

$$
\mathcal{L}_{X} \operatorname{Ric}=\mathcal{L}_{X}(\lambda-\psi+\rho R) g
$$

and since $(\lambda-\psi), \rho$ and $R$ are all constant, so we get

$$
\mathcal{L}_{X} \operatorname{Ric}=2(\lambda-\psi+\rho R) \psi g .
$$

Now we can apply Theorem 4.2 of [Yan70, pg. 54], to conclude that $M$ is isometric to a Euclidean sphere.

We proceed to the proof of Theorem 1.8.

Proof. We see from (2.15) of Lemma 2.4 that

$$
\begin{aligned}
\frac{(1-n \rho)}{2} \Delta|X|^{2}= & (1-n \rho)|\nabla X|^{2}+(n \rho-1) \operatorname{Ric}(X, X)+n \rho \nabla_{X} \operatorname{div} X \\
& +2 \rho(1-n \rho) g(\nabla R, X)-(n(2 \rho+1)-2) g(\nabla \lambda, X) .
\end{aligned}
$$

Integrating above over compact $M$, we get

$$
\begin{array}{r}
0=\int_{M}\left[(1-n \rho)|\nabla X|^{2}+(n \rho-1) \operatorname{Ric}(X, X)+n \rho \nabla_{X} \operatorname{div} X\right. \\
\quad+2 \rho(1-n \rho) g(\nabla R, X)-(n(2 \rho+1)-2) g(\nabla \lambda, X)] d v .
\end{array}
$$

Since $\rho \neq \frac{1}{n}$, we get

$$
\begin{gathered}
\int_{M}|\nabla X|^{2} d v=\int_{M}\left[\operatorname{Ric}(X, X)+\frac{n \rho}{n \rho-1} \nabla_{X} \operatorname{div} X-2 \rho g(\nabla R, X)\right. \\
\left.-\frac{(n(2 \rho+1)-2)}{n \rho-1} g(\nabla \lambda, X)\right] d v
\end{gathered}
$$

so if (1.6) holds, then $|\nabla X|^{2}=0$ and hence $X$ is a Killing vector field. Thus, $\left(M^{n}, g, X, \lambda, \rho\right)$ is trivial.

The proof of Corollary 1.9 is a special case of the proof of Theorem 1.8, where we use (2.22) of Remark 2.6. Next, we prove Theorem 1.11 which we restate here.

Theorem 3.5. Let $\left(M^{n}, g, \nabla f, \lambda, \rho\right)$ be a compact gradient $R B$ almost soliton. Then

and

$$
\int_{M}\left|\nabla^{2} f-\frac{\Delta f}{n} g\right|^{2} d v=\frac{(n-2)}{2 n} \int_{M} g(\nabla R, \nabla f) d v
$$

$$
\int_{M}\left|R i c-\frac{R}{n} g\right|^{2} d v=\frac{(n-2)}{2 n} \int_{M} g(\nabla R, \nabla f) d v .
$$

Proof. For proving (3.12), we take the divergence of (2.4) of Proposition 2.1 to get

$$
\begin{aligned}
(1-2 \rho(n-1)) \Delta R+\Delta|\nabla f|^{2}-2(n-1) \Delta \lambda= & 2 \rho g(\nabla R, \nabla f)+2 g(\nabla \lambda, \nabla f) \\
& +(2 \rho R+2 \lambda) \Delta f .
\end{aligned}
$$

By commuting the covariant derivatives, we have

$$
\begin{aligned}
\nabla_{i} \nabla_{i}\left(g\left(\nabla_{j} f, \nabla_{j} f\right)\right) & =2 \nabla_{i}\left(g\left(\nabla_{i} \nabla_{j} f, \nabla_{j} f\right)\right) \\
& =2 g\left(\nabla_{i} \nabla_{i} \nabla_{j} f, \nabla_{j} f\right)+2\left|\nabla^{2} f\right|^{2} \\
& =2 g\left(\nabla_{j} \nabla_{i} \nabla_{i} f-R_{i j i l} \nabla_{l} f, \nabla_{j} f\right)+2\left|\nabla^{2} f\right|^{2} \\
& =2 g(\nabla(\Delta f), \nabla f)+2 \operatorname{Ric}(\nabla f, \nabla f)+2\left|\nabla^{2} f\right|^{2},
\end{aligned}
$$

so (3.14) becomes

$$
\begin{gathered}
(1-2 \rho(n-1)) \Delta R+2 g(\nabla(\Delta f), \nabla f)+2 \operatorname{Ric}(\nabla f, \nabla f)+2\left|\nabla^{2} f\right|^{2}-2(n-1) \Delta \lambda= \\
2 \rho g(\nabla R, \nabla f)+2 g(\nabla \lambda, \nabla f)+(2 \rho R+2 \lambda) \Delta f .
\end{gathered}
$$


From (2.1) of Proposition 2.1, we know that $\Delta f=n \lambda+(n \rho-1) R$, which on differentiation and using (2.5) becomes

$$
\begin{aligned}
0 & =\nabla_{i} \Delta f+(1-n \rho) \nabla_{i} R-n \nabla_{i} \lambda \\
& =(1-n \rho) \nabla_{i} R+\nabla_{j} \nabla_{i} \nabla_{j} f-R_{i l} \nabla_{l} f-n \nabla_{i} \lambda \\
& =(1-n \rho) \nabla_{i} R+\nabla_{j}\left(-R_{i j}+\lambda g_{i j}+\rho R g_{i j}\right)-R_{i l} \nabla_{l} f-n \nabla_{i} \lambda \\
& =\left(\frac{1}{2}-\rho(n-1)\right) \nabla_{i} R-R_{i l} \nabla_{l} f+(1-n) \nabla_{i} \lambda
\end{aligned}
$$

and hence

$$
2 \operatorname{Ric}(\nabla f, \nabla f)=(1-2 \rho(n-1)) g(\nabla R, \nabla f)+2(1-n) g(\nabla \lambda, \nabla f) .
$$

So, using (3.16) and $\Delta f=n \lambda+(n \rho-1) R$, the left hand side of (3.15) becomes

$$
(1-2 \rho(n-1)) \Delta R+2\left|\nabla^{2} f\right|^{2}-2(n-1) \Delta \lambda+2 g(\nabla \lambda, \nabla f)+(2 \rho-1) g(\nabla R, \nabla f)
$$

and hence (3.15) becomes

$$
(1-2 \rho(n-1)) \Delta R+2\left|\nabla^{2} f\right|^{2}-2(n-1) \Delta \lambda=g(\nabla R, \nabla f)+(2 \rho R+2 \lambda) \Delta f .
$$

Since $\left|\nabla^{2} f-\frac{\Delta f}{n} g\right|^{2}=\left|\nabla^{2} f\right|^{2}-\frac{(\Delta f)^{2}}{n},(3.17)$ becomes

$$
\begin{aligned}
(1-2 \rho(n-1)) \Delta R+2\left|\nabla^{2} f-\frac{\Delta f}{n} g\right|^{2}= & g(\nabla R, \nabla f)+(2 \rho R+2 \lambda) \Delta f-2 \frac{(\Delta f)^{2}}{n} \\
& +2(n-1) \Delta \lambda \\
= & g(\nabla R, \nabla f)+(2 \rho R+2 \lambda) \Delta f \\
& -2 \frac{(\Delta f)}{n}(n \lambda+(n \rho-1) R)+2(n-1) \Delta \lambda \\
= & g(\nabla R, \nabla f)+\frac{2}{n} R \Delta f+2(n-1) \Delta \lambda .
\end{aligned}
$$

Integrating (3.18) over compact $M$, we obtain

$$
\begin{aligned}
\int_{M} 2\left|\nabla^{2} f-\frac{\Delta f}{n} g\right|^{2} d v & =\int_{M}\left[g(\nabla R, \nabla f)+\frac{2}{n} R \Delta f\right] d v \\
& =\frac{(n-2)}{n} \int_{M} g(\nabla R, \nabla f) d v,
\end{aligned}
$$

where we have used integration by parts in the first equality to get the second equality. This proves (3.12).

For proving (3.13), note that

$$
\begin{aligned}
\operatorname{Ric}-\frac{R}{n} g & =-\nabla^{2} f+\lambda g+\rho R g-\frac{R}{n} g \\
& =-\nabla^{2} f+\left(\lambda+\rho R-\frac{R}{n}\right) g \\
& =-\nabla^{2} f+\frac{\Delta f}{n} g
\end{aligned}
$$

and then (3.13) follows from (3.12).

Remark 3.6. Since a gradient RB soliton is a special case of a gradient RB almost soliton, the proof of Theorem 1.11, with $\nabla \lambda=0$, shows that the same integral formulas (3.12) and (3.13) hold for a compact gradient RB solitons as well.

Finally, using Theorem 1.11, we prove Corollary 1.12. 
Proof. Observe that any of the assertions of Corollary 1.12 enable us to conclude that the right hand side of (3.13) is less than or equal to zero and hence Ric $=\frac{R}{n} g$. So, from (2.5), we see that

$$
\nabla_{i} \nabla_{j} f=\left(\lambda+R\left(\rho-\frac{1}{n}\right)\right) g
$$

and hence $\nabla f$ is a nontrivial conformal vector field, so from Theorem 1.5, we get that $M^{n}$ is isometric to a Euclidean sphere.

\section{REFERENCES}

[ABR11] C. Aquino, A. Barros, and E. Ribeiro Jr., Some applications of the Hodge-de Rham decomposition to Ricci solitons, Results Math. 60 (2011), no. 1-4, 245-254. MR2836897 22

[Aub70] Thierry Aubin, Métriques riemanniennes et courbure, J. Differential Geometry 4 (1970), 383-424. MR0279731 个1

[Bou81] Jean-Pierre Bourguignon, Ricci curvature and Einstein metrics, Global differential geometry and global analysis (Berlin, 1979), 1981, pp. 42-63. MR636265 个1

[BR12] A. Barros and E. Ribeiro Jr., Some characterizations for compact almost Ricci solitons, Proc. Amer. Math. Soc. 140 (2012), no. 3, 1033-1040. MR2869087 ^2, 3

$\left[\mathrm{CCD}^{+}{ }^{17}\right]$ Giovanni Catino, Laura Cremaschi, Zindine Djadli, Carlo Mantegazza, and Lorenzo Mazzieri, The Ricci-Bourguignon flow, Pacific J. Math. 287 (2017), no. 2, 337-370. MR3632891 个1

[CM16] Giovanni Catino and Lorenzo Mazzieri, Gradient Einstein solitons, Nonlinear Anal. 132 (2016), 66-94. MR3433954 $\uparrow 2$,

[CMM15] Giovanni Catino, Lorenzo Mazzieri, and Samuele Mongodi, Rigidity of gradient Einstein shrinkers, Commun. Contemp. Math. 17 (2015), no. 6, 1550046, 18. MR3485875 ^2, 4

[Lic58] André Lichnerowicz, Géométrie des groupes de transformations, Travaux et Recherches Mathématiques, III. Dunod, Paris, 1958. MR0124009 $\uparrow 7$

[Oba62] Morio Obata, Certain conditions for a Riemannian manifold to be isometric with a sphere, J. Math. Soc. Japan 14 (1962), 333-340. MR0142086 个7

[PRRS11] Stefano Pigola, Marco Rigoli, Michele Rimoldi, and Alberto G. Setti, Ricci almost solitons, Ann. Sc. Norm. Super. Pisa Cl. Sci. (5) 10 (2011), no. 4, 757-799. MR2932893 个2, 3

[PW09] Peter Petersen and William Wylie, Rigidity of gradient Ricci solitons, Pacific J. Math. 241 (2009), no. 2, 329-345. MR2507581 $\uparrow 3,5$

[Tas65] Yoshihiro Tashiro, Complete Riemannian manifolds and some vector fields, Trans. Amer. Math. Soc. 117 (1965), 251275. MR0174022 $\uparrow 7$

[Yan70] Kentaro Yano, Integral formulas in Riemannian geometry, Pure and Applied Mathematics, No. 1, Marcel Dekker, Inc., New York, 1970. MR0284950 个8

Department of Pure Mathematics, University of Waterloo, Waterloo, ON N2L3G1

E-mail address: s2dwived@uwaterloo.ca 